\title{
Clarification of a Decoupling Method for Multiconductor Transmission Lines ${ }^{*}$ \\ F. Szidarovszky and O. A. Palusinski \\ University of Arizona \\ Tucson, AZ 85721
}

\section{Introduction}

Design of Printed Wire Boards (PWB) for high speed circuits requires careful analysis of signal transmission problems. Such analysis is based on electrical models of interconnections in the form of transmission lines. Models are simplified to lossless transmission lines because they are relatively easy to analyze and they depict adequately such phenomena as cross-talk or reflections at the terminations. The only parameters that are underestimated by lossless lines are signal delay and dispersion. These effects cannot be adequately analyzed using lossless transmission lines. However, cross-talk and reflections are very important in signal integrity considerations and there are several papers dealing with analyses of multiple-coupled, lossless transmission lines. Such analysis involves some intricate aspects of numerical linear algebra, which are not typically known. A recent paper by Lei et al. (1995) attempts to describe and systematize details of such analysis. However, further modifications yielding very efficient and stable numerical procedures for calculating characteristic admittance or impedance matrices and transmission system eigenvalues are possible. Such calculations are performed commonly in software packages supporting design of interconnections and thus their numerical efficiency is important. These useful modifications and simplifications to algorithms proposed in the referenced paper are described in this note. They are easy to implement in existing programs.

\section{Problem formulation}

In their interesting paper, Lei et al (1995) have suggested a new transformation method for solving the matrix telegrapher's equations

$$
\begin{aligned}
& \frac{d}{d z} \underline{V}=-j \omega \underline{L} \underline{I} \\
& \frac{d}{d z} \underline{I}=-j \omega \underline{K} \underline{V} .
\end{aligned}
$$

where, $\underline{V}, \underline{I}$ are vectors of line voltages and currents, $Z$ is the distance along the lines, $j=\sqrt{-1}, \omega$ is the angular frequency and $L, K$ are symmetric, positive definite.

\footnotetext{
${ }^{*}$ Comments concerning the paper: G.-T. Lei, G.-W. Pan, and B. K. Gilbert, “Examination, Clarification and Specification of Model Decoupling Method for Multiconductor Transmission Lines," IEEE Trans. Microwave Theory Tech., vol. 43, no. 9, pp. 2090-2100, Sept. 1995.
} 
The method is based on the simultaneous diagonalization of matrices $\underline{L}$ and $\underline{K}$ by congruence transformation, and the authors show that system (1)-(2) can be reduced to a set of independent single-dimensional equations. In addition, a special matrix transformation can be applied to find the characteristic admittance and impedance matrices.

\section{Decoupling via similarity transformation}

We would like to point out that similarity transformations are easier to use, and their theory is well developed. Furthermore, the diagonalization of symmetric matrices is based on orthogonal transformations, which are numerically very stable (see, for example, Szidarovszky and Yakowitz, 1978). Consequently, from both theoretical and practical points of view it is important to utilize all properties of telegraphers equations and recast the idea of Lei et al (1995) in terms of similarity matrix transformations.

Notice first that,

$\underline{L} \underline{K}=\underline{L}^{\frac{1}{2}}\left(\underline{L}^{\frac{1}{2}} K \underline{L^{\frac{1}{2}}}\right) \underline{L}^{-\frac{1}{2}}$, where both matrices $\underline{L}$ and $\underline{K}$ are symmetric and positive definite.

Since $\underline{L}^{\frac{1}{2}} \underline{K} \underline{L}^{\frac{1}{2}}$ is symmetric and positive definite, there exists an orthogonal matrix $Q$ (such that $\underline{Q}^{-1}=Q^{T}$ ), and a diagonal matrix $\underline{D}$ with positive diagonal such that $\underline{L}^{\frac{1}{2}} \underline{K} \underline{L}^{\frac{1}{2}}=\underline{Q}^{T} \underline{D} \underline{Q}$, and therefore

$$
\underline{L} \underline{K}=\left(\underline{L}^{\frac{1}{2}} \underline{Q}^{T}\right) \underline{D}\left(\underline{Q} \underline{L}^{-\frac{1}{2}}\right)
$$

Using the property of $Q$ matrix the following relation can be obtained

$$
\left(\underline{Q}^{-\frac{1}{2}}\right)^{-1}=\underline{L}^{\frac{1}{2}} \underline{Q}^{-1}=\underline{L}^{\frac{1}{2}} \underline{Q}^{T}
$$

which in turn yields the expression for the square root of $L K$ matrix

$$
\sqrt{\underline{L} \underline{K}}=\left(\underline{L}^{\frac{1}{2}} \underline{Q}^{T}\right) \sqrt{\underline{D}}\left(\underline{Q} \underline{L}^{-\frac{1}{2}}\right)
$$

where $\sqrt{D}$ is obtained from $\underline{D}$ by replacing the diagonal elements by their positive square roots. Therefore the characteristic admittance matrix (Lei et al. 1995 and Reiss and Palusinski 1996) can be written as

$$
\underline{Y}_{o}=\underline{L}^{-1} \sqrt{\underline{L}} \underline{K}=\left(\underline{L}^{-\frac{1}{2}} \underline{Q}^{T}\right) \sqrt{\underline{D}}\left(\underline{Q} \underline{L}^{-\frac{1}{2}}\right)
$$


Notice that $\sqrt{\underline{D}}$ is a diagonal matrix with positive diagonal elements, and $\left(\underline{Q} \underline{L}^{-\frac{1}{2}}\right)^{T}=\underline{L}^{-\frac{1}{2}} \underline{Q}^{T}$, which imply that $\underline{Y}_{o}$ is a positive definite symmetric matrix, which can be easily computed from the diagonal form (3) of matrix $\underline{L} \underline{K}$. Similarly, the characteristic impedance matrix is obtained by using the following formula:

$\underline{Z}_{o}=\underline{Y}_{o}^{-1}=\left(\underline{L}^{-\frac{1}{2}} \underline{Q}^{T}\right) \underline{D}^{-\frac{1}{2}}\left(\underline{Q}^{-\frac{1}{2}}\right)$

which is also very easy to compute.

The above derivation implies the following facts:

(i) Matrix $\underline{L} \underline{K}$ can be diagonalized by similarity transformation, all eigenvalues of $\underline{L} \underline{K}$ are positive, furthermore the eigenvectors $\underline{u}_{i}(i=1,2, \ldots n)$ can be normalized such that for all $i$ and $j$,

$\underline{u}_{i}^{T} \underline{L}_{\underline{u}}=\delta_{i j}=\left\{\begin{array}{lll}1 & \text { if } & i=j \\ 0 & \text { if } & i \neq j\end{array}\right.$

(ii) $\underline{Y}_{o}$ and $\underline{Z}_{o}$ can be obtained easily from the spectral factorization (3) of matrix $\underline{L} \underline{K}$.

(iii) Both matrices $\underline{Y}_{o}$ and $\underline{Z}_{o}$ are symmetric, and positive definite.

The computation can be done by two different ways:

(a) Find the spectral factorization (3) of the non-symmetric matrix $\underline{L} \underline{K}$, and use equations (4) and (5) to find $\underline{Y}_{o}$ and $\underline{Z}_{o}$.

(b) Diagonalize the symmetric positive definite matrices $\underline{L}$ and then $\underline{L}^{\frac{1}{2}} \underline{K} \underline{L}^{\frac{1}{2}}$, which is much easier to perform than factioning non-symmetric matrices, and use (4) and (5) to find $\underline{Y}_{o}$ and $\underline{Z}_{o}$.

\section{Application to the matrix telegrapher's equation}

The diagonal form of $\underline{L} \underline{K}$ enables a decomposition of equations (1) and (2) into a set of single dimensional equations in the following way. Notice first that

$\frac{d^{2}}{d z^{2}} \underline{V}=-j \omega \underline{L} \frac{d}{d z} \underline{I}=-\omega^{2}(\underline{L} \underline{K}) \underline{V}$.

It is known that $\underline{L} \underline{K}=\underline{R} \underline{D} \underline{R}^{-1}$ where $\underline{R}=\underline{L}^{\frac{1}{2}} \underline{Q}^{T}$.

Introducing the new variable 
$\underline{\tilde{V}}=\underline{R}^{-1} \underline{V}$

and using the equation (6) results in

$\frac{d^{2}}{d z^{2}} \underline{\tilde{V}}=\underline{R}^{-1} \frac{d^{2}}{d z^{2}} \underline{V}=-\omega^{2}\left(\underline{R}^{-1} \underline{L} \underline{K} \underline{R}\right) \underline{\tilde{V}}=-\omega^{2} \underline{D} \underline{\tilde{V}}$

This relation shows indeed that the equations in the new variables are independent, since $\underline{D}$ is a diagonal matrix.

\section{Conclusion}

Use of similarly transformation yields more efficient numerical algorithms for computation of eigenvalues and characteristic impedance/admittance matrices of telegrapher's equations than those based on congruence transformation. The algorithms are easy to implement in existing software and thus their utilization is recommended. 


\section{References}

G.-T. Lei, G.-W. Pan, and B. K. Gilbert, " Examination, Clarification and Specification of Model Decoupling Method for Multiconductor Transmission Lines," IEEE Trans. Microwave Theory Tech., vol. 43, no. 9, pp. 2090-2100, Sept. 1995.

K. Reiss and O. A. Palusinski, "Procedure for Direct Calculation of Characteristic Admittance Matrix of Coupled Transmission Lines," IEEE Trans. on Microwave Theory and Techniques, vol. MTT-44, No. 1, January 1996.

F. Szidarovszky and S. Yakowitz, Principles and Procedures of Numerical Analysis, New York: Plenum, 1978. 